\title{
La educación para el desarrollo sostenible en la educación superior: el caso del programa PROFACAD de la universidad de Guadalajara
}

\author{
Oscar Zuñiga Sánchez \\ zunigaoscar777@gmail.com \\ https://orcid.org/0000-0003-2805-1961 \\ Universidad de Guadalajara \\ Luis Carlos Durand Moreno \\ Luis.durand@cutonala.udg.mx \\ Universidad de Guadalajara \\ Espicio Monteros Curiel \\ espicio3@yahoo.com \\ Universidad de Guadalajara
}

\section{RESUMEN}

La Educación para el Desarrollo Sostenible es un proceso en el cual, los docentes juegan un papel importante en la formación de las competencias sustentables en el estudiantado. Sin embargo, esto implica que la gestión universitaria desarrolle un proceso continuo de formación docente acorde con el enfoque de la EDS. Mediante el estudio de caso, se analizó el programa de PROFACAD (Programa de Formación, Actualización y Capacitación Docente) implementado por la Universidad de Guadalajara, como respuesta estratégica para desarrollar en los docentes las características, actitudes, habilidades y conocimientos coherente con el desarrollo sostenible. Se concluye la implicación para la gestión universitaria es establecer estos mecanismos que no se limiten en la formación, sino también en la evaluación del impacto de tiene el Programa de formación docente en el proceso de docencia y desde la perspectiva del estudiantado.

Palabras Clave: gestión educativa; docente; educación ambiental; universidad 


\title{
Education for sustainable development in higher education: the case of the PROFACAD program at the university of Guadalajara
}

\begin{abstract}
Education for Sustainable Development is a process in which teachers play an important role in the formation of sustainable competencies in the student body. However, this implies that university management develops a continuous process of teacher training according to the ESD approach. Through the case study, the PROFACAD (Teacher Training, Updating and Training Program) program implemented by the University of Guadalajara was analyzed, as a strategic response to develop in teachers the characteristics, attitudes, skills, and knowledge according to sustainable development. The implication for university management is to establish these mechanisms that are not limited to training, but also to the evaluation of the impact of the teacher training program on the teaching process and from the perspective of the student body.
\end{abstract}

Keywords: educational management; teachers; environmental education; university

Artículo recibido: 30 noviembre. 2021 Aceptado para publicación: 29 diciembre 2021 Correspondencia: zunigaoscar777@gmail.com

Conflictos de Interés: Ninguna que declarar 


\section{INTRODUCCIÓN}

De acuerdo con la Agenda 2030 de los objetivos para el Desarrollo Sostenible, la educación superior es clave para la formación de una ciudadanía global a efecto de que, en su práctica cotidiana y profesional, pueda contribuir al desarrollo económico, social y ambiental. Para esto, las universidades deben establecer las políticas y mecanismos de gestión para lograr una calidad educativa que contemple el enfoque de la Educación para el Desarrollo Sostenible -EDS-.

De acuerdo con el objetivo 4, de la Agenda 2030, refiere a la importancia de asegurar una educación de calidad que sea inclusiva y con la equidad para promover las oportunidades de aprendizaje a lo largo de la vida para todos (Naciones Unidas, 2016). Asimismo, como parte de la meta 4.c plantea "para el 2030, aumentar considerablemente la oferta de docentes calificados, incluso mediante la cooperación internacional para la formación de docentes en los países en desarrollo, especialmente los países menos adelantados y los pequeños estados insulares en desarrollo" (UNESCO, 2016, p. 38).

La EDS concebida como un proceso contempla que las y los docentes adquieran el conocimiento y las habilidades requeridas por el Desarrollo Sostenible (DS), particularmente desarrollen las competencias claves sustentables (Gradilla, 2017) con el fin de aplicar en su práctica necesaria para formar estudiantes como agentes de cambio (Leal Filho et al., 2017). No obstante, la tarea no es fácil como se ha señalado en otras investigaciones por cuestiones de factores relacionados con la gestión universitaria (Blanco-Portela et al., 2017; González et al., 2015; Lozano et al., 2013).

Por su parte, Zúñiga (2021) advierte que el reto para las universidades públicas en México implica en conocer la forma en que son entendida y aplicada las el concepto de la EDS por los distintos actores de la comunidad universitaria. Para lograr implementar un aprendizaje basado en la EDS se requiere contemplar un enfoque sistémico (Martínez-

Fernández \& González, 2015) y una pedagogía que permita desarrollar las competencias en materia de sostenibilidad en el profesorado.

Los docentes son actores claves en la enseñanza y el aprendizaje de los estudiantes para transmitir de manera favorable los conocimientos disciplinares y transversales, así como desarrollar en los estudiantes las actitudes favorables hacia las cuestiones del Desarrollo sostenible (Biasutti et al., 2018). Contar con profesores formados y capacitados es parte de los rasgos que caracterizan una educación de calidad (Marúm et al., 2015; Olaskoaga, 
2009; UNESCO, 2016). De acuerdo con esto, la universidad está obligada a dirigir sus esfuerzos para lograr establecer políticas que permitan alcanzar su objetivo principal: establecer un proceso de EDS transversal a su quehacer universitario. La formación de actitudes positivas en los docentes respecto al DS es una de las necesidades fundamentales para alcanzar su objetivo (Biasutti et al., 2018).

De acuerdo con esto, para efecto de dirigir el presente ensayo partimos de la siguiente pregunta ¿Qué implicaciones tiene para la gestión formar a las y los docentes con los conocimientos y actitudes en favor del desarrollo sostenible? El objetivo de este artículo es describir la política implementada por la Universidad de Guadalajara para formar al profesorado sobre los principios y metodologías que comprende en el enfoque de la EDS como parte de su compromiso con la Agenda 2030 de los Objetivos para el Desarrollo Sostenible, y su implicación para la gestión universitaria.

\subsection{La Educación para el Desarrollo Sostenible en la educación superior}

Parte de la estrategia para desarrollar un proceso de la enseñanza del desarrollo sostenible es la inaplazable necesidad de formar a las y los docentes para aumentar sus oportunidades de lograr el desarrollo de las actitudes y comportamientos en el estudiantado con los principios que demanda el DS. De acuerdo con Lozano (2014) educar a los educadores representa un elemento importante para promover la incorporación de una EDS en las universidades.

Por su parte, Arbuthnott (2009) afirma que la mayoría de los programas de EDS tienen como objetivo cambiar las actitudes y valores de los ciudadanos con su entorno natural. Sin embargo, argumenta que tanto los programas como los proyectos relacionados con la EDS deben estar dirigidos hacia el control de los factores contextuales que facilitan $u$ obstaculizan este proceso. De acuerdo con esto, consideramos que factores como las disciplinas en las cuales, el profesor se desarrolla en su ámbito profesional, puede ser un factor que pudiera facilitar o impedir la enseñanza de la EDS en estudiantes de pregrado. Por ejemplo, no es igual para un docente que se desarrolla en el campo de la biología y la ecología, la enseñanza de los principios del DS, como lo haría uno(a) docente que se desarrolla en el campo de la economía o las humanidades.

Por su parte, Tang (2018) sostiene que los cursos especializados en el aprendizaje del desarrollo sostenible genera impactos positivos en las creencias, actitudes e intenciones de quienes lo estudian y practican. De acuerdo con Aznar et al. (2011) y Biasutti et al. 
(2018), la capacitación de los docentes para aplicar la sustentabilidad en su práctica docente no sólo es trascendental, sino también, se conviertan en agentes del cambio al transmitir los principios del DS en los estudiantes en formación.

Si bien, los programas relacionados con la formación de competencias y actitudes positivas hacia el DS son de gran importancia, algunas veces no contemplan de manera completa sus objetivos. De acuerdo con Keles (2017), la EDS no se práctica ampliamente lo que produce una brecha entre las intenciones y lo que realmente se practica en el proceso de formación. Argumenta que los docentes no comprenden bien el concepto de la EDS y la forma de cómo practicarlos, por lo que recurren a prácticas tradicionales. Asimismo, argumenta que la EDS apoya otras formas de pedagogías más transformadoras centradas en el estudiante para fomentar un aprendizaje activo, participativo y experiencial (Keles, 2017).

De acuerdo con Christie, Miller, Cooke y White (2015) en estudios previos se han encontrado que las percepciones académicas respecto a la educación para la sostenibilidad tiene un impacto significativo como inhibidor de la inclusión de estos propósitos en el currículum y que por lo tanto si está comprendido en el propósito, entonces los docentes deben apoyar y comprender de manera clara el concepto de sostenibilidad de tal modo que no afecte negativamente en el aprendizaje de los estudiantes. Además, señalan que la incorporación de la EDS a la educación superior ha sido más lenta de lo esperado, esto debido a que la propia controversia y dificultad que existe entre los distintos actores para comprender y definir el concepto de sostenibilidad. Otro factor que se relaciona con la reticencia de los profesores para transmitir los contenidos relacionados con las cuestiones del DS a los estudiantes. Otro inconveniente es debido a la manera del cómo se organiza o estructura el currículum universitario, si éste contribuye a una formación disciplinar, y en donde las cuestiones del DS son irrelevantes en algunas disciplinas (Christie et al., 2015).

La EDS se refiere a programas y experiencias educativas que están diseñadas para permitir que las personas adquieran los conocimientos, las habilidades y los valores necesarios para forjar un futuro sostenible (Biasutti \& Frate, 2017). Por lo tanto, existe la necesidad de contar con herramientas y medidas para evaluar el desarrollo de la EDS en las universidades como parte del establecimiento de políticas, programas y sistemas educativos. 
Christie et. al. (2015) por su parte, realizaron un estudio tomando una muestra amplia de docentes en las universidades de Australia para analizar las actitudes de los docentes respecto a la Educación para la Sustentabilidad -por sus siglas en inglés: EfS- con el propósito de determinar sus concepciones y actitudes hacia el concepto, así como, las dificultades que presentan para incorporarla en su práctica docente. Si bien señalan, algunos estudios han evidenciado que no existe diferencias significativas en las concepciones y actitudes sustentables respecto a la disciplina o área de conocimiento, no obstante, advierten que estos estudios son sesgados puesto que trabajan con muestras no significativas reduciéndose a estudio de caso. De acuerdo con esto último, Christie et. al. (2015) tampoco encontró diferencias significativas respecto a las concepciones y actitudes a pesar de que tomó una muestra representativa. No obstante, si las hubo en respecto de las dificultades presentadas en su práctica docente, así como también en las actitudes.

Como parte de sus conclusiones, Christie et al. (2015) afirma que, aunque los académicos no tienen opiniones concretas sobre las concepciones de EfS, no obstante, la mayoría de los académicos docentes en Australia sí respaldan la inclusión de EfS en la enseñanza y en general en las universidades. Asimismo, señalan que estas opiniones todavía pueden ser influenciadas por la disciplina y, por tanto, investigaciones futuras deberían profundizar en estos matices entre las disciplinas. Asimismo, sus estudios evidencian que tanto las ciencias creativas como las ciencias sociales son las dos áreas de conocimiento que en menor medida emplean una pedagogía en la cual se sustenta la Educación para el Desarrollo sostenible.

Por otro lado, los estudios de Aziz et al. (2012) demuestran que entre el conocimiento y la actitud existe una correlación fuerte en relación con las cuestiones del Desarrollo Sustentable. Los autores desarrollan la propuesta de un Modelo de Ecuación Estructural que mide la relación entre conocimiento y la actitud de los estudiantes respecto a las cuestiones de la sustentabilidad.

Por su parte, Christie et. al (2015) advierten que a pesar de que existen numerosos estudios que demuestran y analizan los esfuerzos realizados por las universidades en materia de una educación para el desarrollo sostenible. No obstante, señalan que se enfocan en ofrecer una visión general tomando en cuenta aspectos relacionados con el currículo y en la gestión verde del campus, en lugar de buscar las opiniones y prácticas empíricas 
actuales de los académicos quienes no siempre participan directamente están involucrados en el proceso de la EDS.

De acuerdo con resultados de otros estudios realizados respecto a las opiniones de los docentes se ha encontrado que cuentan con algunas visiones distintas del concepto y también encuentran que los académicos perciben como irrelevante en su disciplina las temáticas relacionadas con el desarrollo sostenible (Christie et al., 2015). Por lo tanto, el estudio realizado por Christie et al. Se basó en analizar una muestra más amplia para ver si existen diferencias significativas entre las opiniones de los docentes respecto a su disciplina en la que se desarrollan y sí también existe diferencia entre las opiniones respecto del campus de adscripción

Park et al. (2016) por su parte investigaron las comprensiones y las actitudes de los docentes coreanos de una primaria Koreana. Como parte de sus hallazgos, encuentra que la mayoría había accedido a información sobre desarrollo sostenible y EDS mediante talleres, seminarios o capacitación. Sin embargo, solo unos pocos -14\%- entendieron el concepto de desarrollo sostenible o ESD bastante bien o muy bien. Si bien los resultados demuestran que los docentes mostraron actitudes positivas hacia el desarrollo sostenible o la EDS, pero carecían de una correcta comprensión del concepto.

\section{ESTRATEGIA METODOLÓGICA}

Para esta investigación se consideró emplear un método mixto como es el estudio de caso y el análisis y síntesis de fuentes documentales generados por la instancia oficial de la Universidad.

\section{El caso de la Benemérita Universidad de Guadalajara}

La Universidad de Guadalajara es la segunda universidad más importante de México, después de la Universidad Autónoma de México. Actualmente tiene presencia en todas las regiones del estado con cobertura en educación media superior y superior. En el año 1994-1995 La Universidad de Guadalajara sufre una transformación tanto en lo normativo como en lo organizacional para convertirse en una red universitaria estructurada de la siguiente forma (Universidad de Guadalajara, 2014): Seis Centro Universitarios temáticos o facultades, nueve Centros Universitarios multidisciplinares, un sistema de Universidad Virtual, un Sistema de Educación Media Superior y una administración general. 
La Universidad atendió para el periodo 2016-2017 al 46\% de estudiantes en pregrado en todo el Estado de Jalisco. Para el mismo periodo se contaba con una población estudiantil a nivel pregrado de 112,335, de los cuales, el $48 \%$ correspondían a hombres, y el $52 \%$ a mujeres. De esta población 101,354, estudiantes de pregrado se encontraban inscritos en algún programas educativo reconocido por su calidad (Universidad de Guadalajara, 2017).

El número de programas ofertados para el 2016 en el nivel de licenciatura y nivelaciones - incluyendo los Técnico Superior Universitario-, 145 programas fueron evaluados por CIEES. En la tabla 1 se muestra la distribución de las licenciaturas ofertadas por la Universidad.

Tabla 1. Distribución de licenciaturas por área del conocimiento

\begin{tabular}{lcc}
\hline Área de conocimiento & $\mathbf{2 0 1 7}$ & $\mathbf{2 0 1 9}$ \\
\hline Ciencias Sociales y Administrativas & 97 & 102 \\
\hline Ingeniería y Tecnología & 53 & 58 \\
\hline Ciencias de la Salud & 28 & 29 \\
\hline Educación y humanidades & 24 & 31 \\
\hline Ciencias Agropecuarias & 8 & 8 \\
\hline Ciencias Naturales y Exactas & 14 & 14 \\
\hline Total de licenciaturas & $\mathbf{2 2 4}$ & $\mathbf{2 4 2}$ \\
\hline
\end{tabular}

Fuente: Elaboración propia con información de anexo estadístico, (2020)

Como parte de su plantilla académica, para el año 2019 la Universidad contaba con 11,478 académicos que ocupan distintos tipos contractuales distribuidos en los Centro Universitarios y el SUV, de los cuales 1,819 también hacen actividades de investigación. En la tabla 1 se muestra la distribución de académicos por Centro Universitario y SUV para el año 2017 (Universidad de Guadalajara, 2020)

Tabla 2. Distribución de académicos por tipo de contrato en el año 2017

\begin{tabular}{|c|c|c|}
\hline Entidad & Docente de carrera* & Docente de asignatura \\
\hline CUAAD & 248 & 434 \\
\hline CUCBA & 342 & 187 \\
\hline CUCEA & 471 & 611 \\
\hline CUCEI & 573 & 552 \\
\hline CUCS & 581 & 1248 \\
\hline CUCSH & 696 & 439 \\
\hline CUTONALÁ & 129 & 400 \\
\hline CUALTOS & 104 & 231 \\
\hline CUCIENEGA & 181 & 324 \\
\hline
\end{tabular}




\begin{tabular}{lcc} 
CUCOSTA & 139 & 359 \\
\hline CUCOSTASUR & 170 & 195 \\
\hline CUSUR & 130 & 419 \\
\hline CULAGOS & 86 & 160 \\
\hline CUNORTE & 72 & 337 \\
\hline CUVALLES & 69 & 167 \\
\hline SUV & 92 & 374 \\
\hline subtotal & $\mathbf{2 3 4 0}$ & $\mathbf{6 4 3 7}$ \\
\hline
\end{tabular}

*Se incluyen profesores de tiempo completo y medio tiempo

Fuente: Elaboración propia con base en la información de anexo estadístico (2020).

La Universidad se ha comprometido para atender los problemas que impiden el tránsito hacia un desarrollo sostenible y sustentable, como así fue establecido en su misión el año 2014, “[...] las necesidades educativas de nivel medio superior y superior con calidad y pertinencia. Promueve la investigación científica y tecnológica, así como la vinculación y extensión para incidir en el desarrollo sustentable e incluyente de la sociedad." (Universidad de Guadalajara, 2014, p. 51). Para ello, la institución estableció seis ejes estratégicos para el cumplimiento de sus objetivos generales, de los cuales se destaca aquel que refiere: Universidad sustentable y socialmente responsable. Directrices que también son transversales a cinco ejes temáticos: Docencia y aprendizaje, investigación y posgrado, vinculación, extensión y difusión, internacionalización, gestión y gobierno (Universidad de Guadalajara, 2014). Este dato resulta de interés dado que el programa de PROFACAD se diseñó y desarrolló en los sucesivo.

Como parte de los objetivos relacionadas con el eje de Docencia y aprendizaje se establece que: "Los profesores deben desarrollar un perfil que les permita hacer frente a las nuevas necesidades de formación [...]" (UdeG,2014, p.6). Asimismo, en el objetivo 3 , se establece como parte de las estrategias, crear un programa integral para la formación y actualización de las actividades docentes con el propósito de consolidar enfoques pedagógicos acordes con el modelo de un aprendizaje centrado en el estudiante (Universidad de Guadalajara, 2014).

\section{Programa de PROFACAD: una política institucional para la actualización docente}

El Programa de Formación, Actualización y Capacitación Docente -PROFACAD- tuvo como objetivo actualizar a la planta docente con los conocimientos necesarios para proponer mejoras al diseño curricular, así como la adopción de mejores prácticas docentes en el proceso de formación académica. Esta política destacaba la importancia del 
profesorado como sujeto clave en el proceso de enseñanza y aprendizaje (CGA-UdeG, 2018).

Este Programa intentó cerrar una brecha identificada por la Institución, de las exigencias y demandas de la sociedad para ofrecer una educación superior acorde con el siglo XXI. Para ello, se estructuró inicialmente en cinco módulos que contemplaron 22 talleres. Se pretendió que cada módulo fortaleciera una dimensión del proceso formativo docente.

Imagen 1. Estructura del Programa PROFACAD

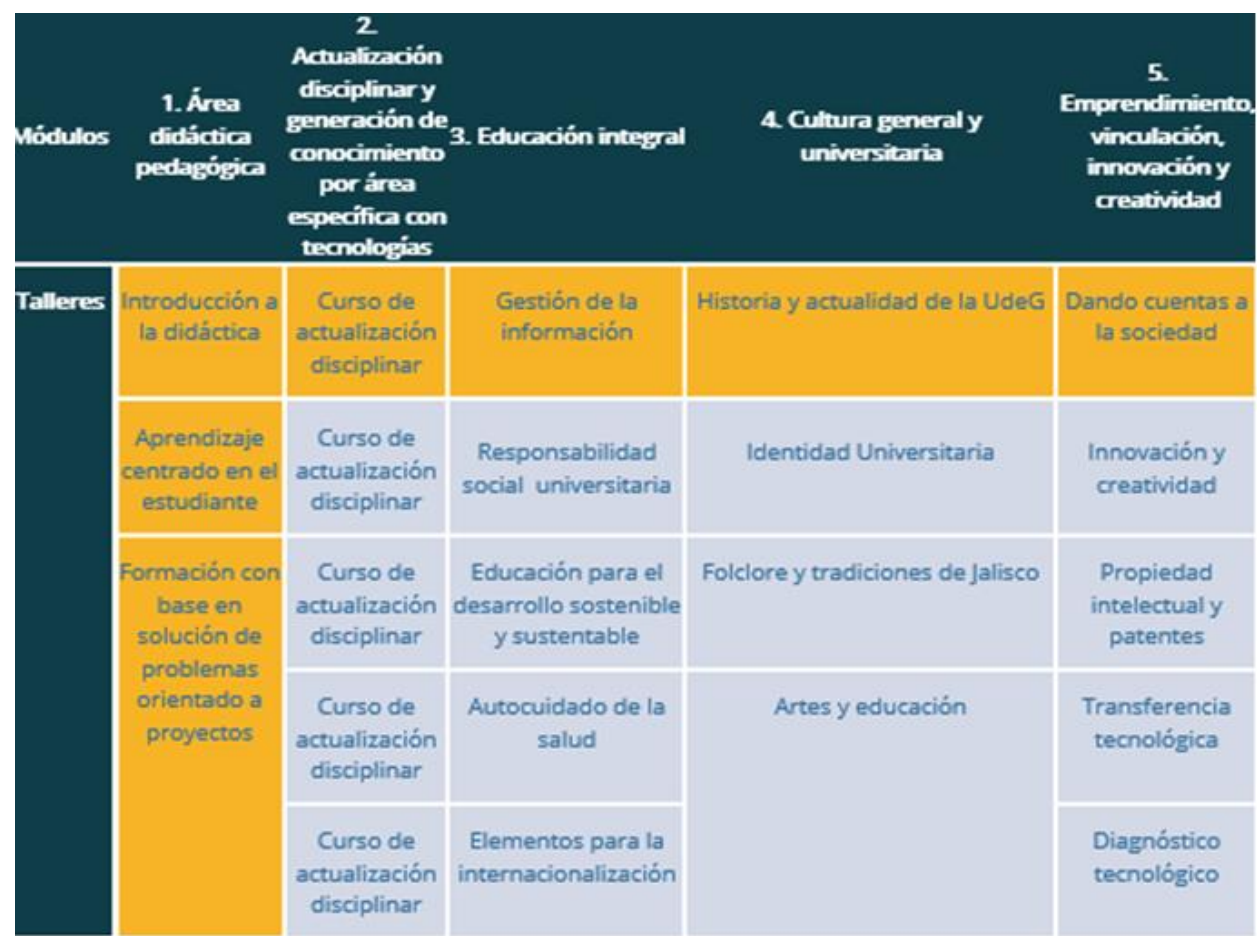

Taller obligatorio Taller optativo

Fuente: Coordinación General Académica, (2018)

Como parte de la estrategia, cada dependencia de la Red Universitaria contaba con una persona responsable de coordinar los talleres, sin embargo, la instancia responsable de la ejecución de esta política fue la Coordinación General Académica a través de su Unidad de Mejoramiento y Formación Académica (UMEFORA), misma que concentraba las solicitudes realizadas por las dependencias de la Red.

En lo que refiere particularmente al módulo tres, que corresponde a Educación Integral, se ubicó el taller de Educación para el Desarrollo Sostenible y Sustentable. Como parte de las expectativas del taller, se esperaba que los docentes desarrollen las actitudes, 
habilidades y conocimientos para que lo fomenten en sus estudiantes, acorde con las demandas de la Agenda 2030 y del desarrollo sostenible. Estas habilidades son, entre otras: un pensamiento crítico y reflexivo, facilitar las experiencias de aprendizaje significativo en sus estudiantes, promover proyectos didácticos, planificar los procesos de enseñanza y aprendizaje centrados en el estudiante y ubicar los problemas que enfrenta el desarrollo sostenible en el currículo universitario contemplando todas las disciplinas.

Tabla 3: Talleres impartidos en el periodo 2015-2017

\begin{tabular}{lcccc}
\hline \multirow{2}{*}{ DEPENDENCIA } & & periodo & & subtotal \\
\cline { 5 - 5 } & $\mathbf{2 0 1 5}$ & $\mathbf{2 0 1 6}$ & $\mathbf{2 0 1 7}$ & \\
\hline CUAAD & 14 & 13 & 16 & 43 \\
\hline CUCBA & 6 & 0 & 7 & 13 \\
\hline CUCEA & 4 & 16 & 6 & 26 \\
\hline CUCEI & 0 & 49 & 50 & 99 \\
\hline CUCS & 64 & 54 & 63 & 181 \\
\hline CUCSH & 12 & 18 & 13 & 43 \\
\hline CUTONALÁ & 5 & 11 & 15 & 31 \\
\hline CUALTOS & 11 & 13 & 13 & 37 \\
\hline CUCIENEGA & 6 & 14 & 16 & 36 \\
\hline CUCOSTA & 10 & 12 & 14 & 36 \\
\hline CUCOSTASUR & 14 & 11 & 11 & 36 \\
\hline CUSUR & 14 & 17 & 15 & 46 \\
\hline CULAGOS & 3 & 8 & 8 & 19 \\
\hline CUNORTE & 3 & 21 & 0 & 24 \\
\hline CUVALLES & 15 & 5 & 8 & 28 \\
\hline SUV & 2 & 3 & 16 & 21 \\
\hline CGA & 4 & 16 & 5 & 25 \\
\hline Total & 187 & 281 & 276 & 744 \\
\hline
\end{tabular}

Fuente: Coordinación General Académica, (2018)

Cada taller tuvo una duración de 40 hrs. De las cuales, se dividió una parte en presencial y la otra en trabajo independiente y de manera virtual. Con excepción de los talleres del módulo 2, que consistía en la formación y actualización disciplinar acorde con el perfil profesional del profesorado, estos debían ser propuestos de acuerdo con las necesidades de cada dependencia o entidad. Por otro lado, el resto de los talleres fueron liderados por la Coordinación General responsable del Programa. Los instructores que desarrollaban estos talleres eran también, en ocasiones, las y los docentes integrantes de la Universidad. A efecto de que las y los docentes recibieran la acreditación, debían cursar cinco talleres 
que corresponden a cada módulo y con ello poder recibir la certificación de calidad, de acuerdo con los objetivos generales del Programa. Como parte de los entregables, a efecto de acreditar el curso, las y los profesores deberían elaborar su propuesta de desarrollo sostenible aplicado a una unidad de aprendizaje en el contexto de su centro universitario.

Tabla 4. Docentes inscritos a los talleres del programa en el periodo 2015-2017

\begin{tabular}{lccccccc}
\hline \multirow{2}{*}{ Dependencia } & \multicolumn{2}{c}{$\mathbf{2 0 1 5}$} & \multicolumn{2}{c}{$\mathbf{2 0 1 6}$} & \multicolumn{2}{c}{$\mathbf{2 0 1 7}$} & \% \\
& Inscritos & Acrditados & Inscritos & Acreditados & Inscritos & Acreditados & Efectividad \\
\hline CUAAD & 189 & 189 & 215 & 139 & 252 & 223 & 84 \\
\hline CUCBA & 55 & 37 & 0 & 0 & 145 & 139 & 88 \\
\hline CUCEA & 73 & 42 & 325 & 245 & 120 & 100 & 75 \\
\hline CUCEI & 0 & 0 & 1051 & 663 & 1038 & 785 & 69 \\
\hline CUCS & 1120 & 572 & 970 & 471 & 1285 & 778 & 54 \\
\hline CUCSH & 378 & 378 & 318 & - & 393 & 218 & 55 \\
\hline CUTONALA & 67 & 67 & 276 & 182 & 172 & 114 & 70 \\
\hline CUALTOS & 124 & 124 & 251 & 200 & 222 & 184 & 85 \\
\hline CUCIENEGA & 93 & 89 & 286 & 186 & 312 & 254 & 77 \\
\hline CUCOSTA & 221 & 176 & 270 & 66 & 297 & 180 & 54 \\
\hline CUCOSTASUR & 217 & 166 & 156 & 94 & 249 & 158 & 67 \\
\hline CUSUR & 224 & 185 & 376 & 289 & 395 & 267 & 74 \\
\hline CULAGOS & 49 & 43 & 125 & 91 & 171 & 91 & 65 \\
\hline CUNORTE & 63 & 56 & 303 & 278 & 0 & 0 & 91 \\
\hline CUVALLES & 268 & 140 & 63 & 63 & 158 & 128 & 68 \\
\hline SUV & 59 & 23 & 63 & 54 & 425 & 343 & 77 \\
\hline CGA & 53 & 29 & 397 & 308 & 99 & 79 & 76 \\
\hline Total & & & & & & 72 \\
\hline
\end{tabular}

Fuente: Coordinación General Académica, (2018)

De acuerdo con la tabla anterior, se observa que, para el periodo comprendido, en términos generales, la estrategia implementada tuvo una eficacia del $72 \%$ respecto de las y los docentes que lograron concluir satisfactoriamente su proceso de actualización.

\section{RESULTADOS Y DISCUSIÓN}

De acuerdo con Biasutti et al. (2018) los académicos se caracterizan por ser agentes de cambio en la promoción de la EDS en el contexto universitario, de ahí su necesidad para 
adquirir las competencias requeridas e integrar los conceptos de la EDS en sus actividades académicas. No obstante, también las principales barreras en las que enfrenta el profesorado es la falta de capacitación relacionadas con el empleo de las metodologías adecuadas para inculcar los principios del enfoque de la EDS.

El taller de EDSyS tuvo como objetivo particular que el profesorado adquiera los conocimientos, habilidades y valores necesarios para el manejo y aplicación de los conceptos relacionados con el desarrollo sostenible logrando aplicar prácticamente los mismos a situaciones reales en su centro universitario o campus.

El liderazgo que ejerce las y los docentes es una tarea que favorece el desarrollo de un pensamiento sistémico en los estudiantes universitarios. De acuerdo con Zúñiga (2019) Los docentes líderes transformacionales "es aquel visionario que tiene la capacidad de motivar a sus seguidores (estudiantes) para que desarrollen una visión hacia el futuro como parte de las competencias contempladas en la EDS.” (p.93).

Investigaciones (Arbuthnott, 2009; Biasutti et al., 2016) han demostrado que las personas que han sido expuestas a programas o cursos relacionados con la EDS, han demostrado un incremento en las actitudes y compromiso en favor con el medio ambiente. Por otro lado, otros investigadores han encontrado que las actitudes ambientales probablemente se formaron como resultado de experiencias de vida en lugar de la exposición a un curso o programa específico (Ull et al., 2014). En este punto, se mantienen dos argumentos importantes en el mejoramiento de los conocimientos y actitudes de los docentes hacia el DS. A través de programas y mediante el cambio en el contexto y la gestión verde del campus, con todas las implicaciones en la gestión que esto conlleva.

Por otro lado, de acuerdo con Campbell et al. (1999) el conocimiento y las actitudes se correlacionan, es decir, a mayor conocimiento de las personas puede ayudar a mejorar las actitudes hacia las cuestiones medioambientales. No obstante, señalan que también pueden existir influencias externas como el nivel socioeconómico y la cultura como atenuantes en las actitudes de las personas. De acuerdo con esto, aún no se conoce cómo impactó el programa a aquellos docentes que cursaron el taller, de acuerdo con sus características personales y profesionales, situación que pudiera ser una mejora para la gestión universitaria, evaluar la eficacia de esta política institucional.

De acuerdo con la perspectiva de la Responsabilidad Social Universitaria, instaurar el enfoque de la Educación para el Desarrollo Sostenible implica establecer un liderazgo 
institucional a efecto de generar un aprendizaje identificando las buenas prácticas existentes y asegurando los recursos requeridos para ello (Cebrián, 2020)

\section{CONCLUSIÓN}

El programa PROFACAD, representa a la política y la estrategia en la cual la Universidad de Guadalajara demuestra su compromiso en favor con el Desarrollo Sostenible. Un programa en el cual tiene como objetivo la formación integral de los docentes. Tan sólo en el periodo 2015-2017, 9,686 académicos acreditaron debidamente los distintos talleres contemplados por esta política institucional. Sin embargo, las universidades públicas aún tienen el reto de incidir dentro de la práctica docente, una formación de profesionales acorde con el enfoque de la EDS.

El establecimiento de programas de actualización docente para incorporar los principios de la sustentabilidad como parte de la formación integral del estudiantado, es una tarea crucial para la gestión universitaria. Esto implica, no sólo establecer las intenciones en su misión, sino también buscar los mecanismos de evaluación de su impacto en la práctica docente a efecto de identificar puntos de mejora en el proceso.

Es prioritario que la Universidad de Guadalajara mida el impacto de este Programa considerando indicadores cualitativos y desde la perspectiva del estudiantado. Así de esta forma, se tendría mayor certeza sobre el grado de efectividad de la estrategia y, por tanto, la posibilidad de aplicar acciones de mejora para alcanzar un currículum universitario acorde con las demandas del enfoque de la EDS.

\section{REFERENCIAS BIBLIOGRAFICAS}

Arbuthnott, K. D. (2009). Education for sustainable development beyond attitude change. International Journal of Sustainability in Higher Education, 10(2), 152-163.

Aziz, A., Sheikh, S., Yusof, K., Udin, A., \& Yatim, J. (2012). Developing a Structural Model of Assessing Students' Knowledge-Attitudes towards Sustainability. Social and Behavioral Sciences, 56, 513-522.

Aznar Minguet, P., Martinez-Agut, M. P., Palacios, B., Piñero, A., \& U11, M. A. (2011). Introducing sustainability into university curricula: An indicator and baseline survey of the views of university teachers at the University of Valencia. Environmental Education Research, 17(2), 145-166. https://doi.org/10.1080/13504622.2010.502590 
Biasutti, M., De Baz, T., \& Alshawa, H. (2016). Assessing the Infusion of Sustainability Principles into University Curricula. Journal of Teacher Education for Sustainability, 18(2), 21-40.

Biasutti, M., \& Frate, S. (2017). A validity and reliability study of the Attitudes toward Sustainable Development scale. Environmental Education Research, 23(2), 214230.

Biasutti, M., Makrakis, V., Concina, E., \& Frate, S. (2018). Educating academic staff to reorient curricula in ESD. International Journal of Sustainability in Higher Education, 19(1), 179-196. https://doi.org/10.1108/IJSHE-11-2016-0214

Blanco-Portela, N., Benayas, J., Pertierra, L., \& Lozano, R. (2017). Towards the integration of sustainability in Higher Eeducation Institutions: A review of drivers of and barriers to organisational change and their comparison against those found of companies. Journal of Cleaner Production, 166, 563-578.

Cebrián, G. (2020). La educación para el desarrollo sostenible en el currículum universitario: Una investigación-acción cooperativa con profesorado. Revista Iberoamericana de Educación Superior, 11(30), Article 30. https://doi.org/10.22201/iisue.20072872e.2020.30.590

CGA-UdeG. (2018). Programa de Formación, Actualización y Capacitación Docente (PROFACAD). http://www.cga.udg.mx/

Christie, B., Miller, K., Cooke, R., \& White, J. (2015). Environmental sustainability in higher education: What do academics think? Environmental Education Research, 21(5), 655-686. https://doi.org/10.1080/13504622.2013.879697

González, E. J., Meira-Cartea, P. Á., \& Martínez-Fernández, C. N. (2015, julio). Sustentabilidad y Universidad: Retos, ritos y posibles rutas. Revista de la Educación Superior, 44(175), 69-93.

Gradilla, M. (2017). Contribución de las disciplinas académicas al desarrollo de competencias para el desarrollo sostenible en los estudiantes: La perspectiva de los académicos. (Tesis de Doctorado). CUCEA, Universidad de Guadalajara.

Keles, Ö. (2017). Investigation of Pre-Service Science Teachers' Attitudes towards Sustainable Environmental Education. Higher Education Studios, 7(3), 171-180.

Leal Filho, W., Azeiteiro, U., Alvez, F., \& Molthan-Hill, P. (2017). Handbook theory and practice of sustainable Development in Higher Education Vol. 4. Springer. 
Lozano, R., Ceulemans, K., Alonso-Almeida, M., Huisingh, D., Lozano, F., Waas, T., Lambrechts, R., \& Hugé, J. (2014). A review of commitment and implementation of sustainable development in higher education: Results from a world survey. Journal of Cleaner Production, 108, 1-18.

Lozano, R., Lukman, R., Lozano, F. J., Huisingh, D., \& Lambrechts, W. (2013). Declarations for sustainability in higher education: Becoming better leaders, through addressing the university system. Journal of Cleaner Production, 48, 1019. https://doi.org/10.1016/j.jclepro.2011.10.006

Martínez-Fernández, C. N., \& González, E. J. (2015). Las políticas para la sustentabilidad de las instituciones de Educación superior en México: Entre el debate y la acción. Revista de la Educación Superior, 44(174), 61-47.

Marúm, E., Curiel, F., \& Rosario, V. (2015). Estudiantes frente al espejo. Percepciones de la calidad educativa en programas de licenciatura y posgrado (1a ed.). Universidad de Guadalajara.

Naciones Unidas. (2016). Agenda 2030 y los objetivos de desarrollo sostenible. Naciones Unidas- CEPAL.

Olaskoaga, J. (2009). Hacia una educación superior de calidad. Un análisis desde la perspectiva del profesorado en Argentina, Chile, España y México (1a ed.). Universidad Nacional de la Plata.

Park, E., Kim, H., \& Yu, S. (2016). Perceptions and Attitudes of Early Childhood Teachers in Korea About Education for Sustainable Development. International Journal of Early Childhood, 48(3), 369-385. https://doi.org/10.1007/s13158-0160176-y

Tang, K. H. D. (2018). Correlation between sustainability education and engineering students' attitudes towards sustainability. International Journal of Sustainability in Higher Education, 19(3), 459-472. https://doi.org/10.1108/IJSHE-08-20170139

Ull, M. A., Martínez-Agut, M. P., Piñero, A., \& Aznar-Minguet, P. (2014). Perceptions And Attitudes Of Students Of Teacher-Training Towards Environment And Sustainability. Social and Behavioral Sciences, 131, 453-457.

UNESCO. (2016). Desglosar el objetivo de Desarrollo Sostenible 4 Educación 2030. Guía. UNESCO. 
Universidad de Guadalajara. (2014). Plan de desarrollo institucional 2014-2030. Universidad de Guadalajara.

Universidad de Guadalajara. (2017). Estadística institucional 2016-2017. Universidad de Guadalajara.

Universidad de Guadalajara. (2020). Anexo estadístico 2019. Universidad de Guadalajara.

Zúñiga Sánchez, O. (2019). Estilo de liderazgo del profesorado: El caso de la licenciatura en ingeniería en ciencias computacionales del centro universitario de Tonalá, Universidad de Guadalajara. En B. E. Madrigal Torres \& M. Ramírez Mata, La ética y el liderazgo en las instituciones latinoamericanas de educación superior en la cuarta revolución industrial. Fondo Editorial Universitario.

Zúñiga Sánchez, O. (2021). El reto de las universidades públicas de México para incorporar una educación pertinente acorde con la sustentabilidad. RIDE Revista Iberoamericana para la Investigación y el Desarrollo Educativo, 11(22). https://doi.org/10.23913/ride.v11i22.952 